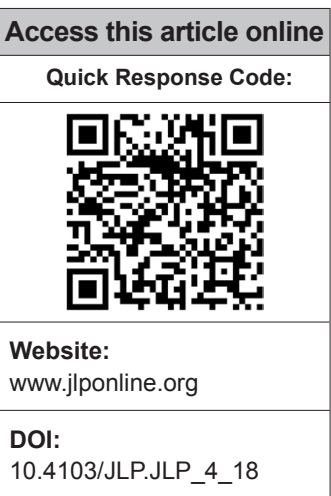

Departments of Pathology and ${ }^{1}$ Microbiology, Andaman and Nicobar Islands Institute of Medical Sciences, Port Blair, Andaman and Nicobar

Address for correspondence:

Dr. Biswajit Dey,

Department of Pathology, Andaman and Nicobar Islands Institute of Medical Sciences,

Port Blair - 744 104, Andaman and Nicobar E-mail: drbish25@ rediffmail.com

Submission: 05-01-2018 Accepted: 08-06-2018

\title{
Tuberculosis cutis orificialis with underlying pulmonary tuberculosis in an immunocompetent man
}

\author{
Biswajit Dey, Archana Hemant Deshpande, Sanjeev Hosdurg Bhat ${ }^{1}$, Ashok Singh
}

\section{Abstract:}

Tuberculosis cutis orificialis (TCO) is a rare form of tuberculosis more often secondary to pulmonary tuberculosis. TCO has varied differential diagnoses and thus results in delayed diagnosis and treatment leading to serious consequences. The diagnosis of TCO is confirmed by biopsy and nucleic acid amplification tests in majority of cases. We report a case of TCO with underlying pulmonary tuberculosis in a 50-year-old male, who presented with a painful nonhealing ulcer of the right buccal mucosa. Biopsy and real-time polymerase chain reaction helped in confirming the diagnosis. The patient was treated with antitubercular therapy.

Key words:

Mycobacterium, oral ulcer, tuberculosis

\section{Introduction}

$\sqcap$ uberculosis cutis orificialis (TCO), a 1 rare form of tuberculosis, constitutes $2 \%$ of cutaneous tuberculosis cases. ${ }^{[1]}$ It usually presents as nonhealing ulcer of the oral cavity. ${ }^{[2]}$ It can be either primary or secondary. ${ }^{[1,2]}$ Secondary tuberculosis orificialis of oral cavity is more often secondary to pulmonary tuberculosis. ${ }^{[3,4]} \mathrm{TCO}$ has varied differential diagnoses. ${ }^{[3]}$ Most of the patients of TCO present with concomitant pulmonary tuberculosis. Thus, an accurate diagnosis may be missed leading to delayed treatment. We report a case of TCO with underlying pulmonary tuberculosis in a 50-year-old male, who presented with a painful nonhealing ulcer of the right buccal mucosa.

\section{Case Report}

A 50-year-old male presented with a painful ulcer in the right oral cavity for 1 month. Intraoral examination revealed a painful

This is an open access journal, and articles are distributed under the terms of the Creative Commons Attribution-NonCommercial-ShareAlike 4.0 License, which allows others to remix, tweak, and build upon the work non-commercially, as long as appropriate credit is given and the new creations are licensed under the identical terms.

For reprints contact: reprints@medknow.com ulcer measuring $5.5 \mathrm{~cm} \times 5 \mathrm{~cm}$ in the right buccal mucosa, and it was extending to the right upper and lower labial mucosa. The right side of the hard palate was also involved. The surface of the ulcer was hyperemic and irregular [Figure 1]. He was a chronic smoker; however, he did not complain of respiratory distress, cough, fever, or hemoptysis. There was no history of dental complaints or trauma. Palpation of the neck nodes revealed a single Level II right cervical lymph node measuring $1.5 \mathrm{~cm}$ in diameter. The laboratory findings showed an elevated erythrocyte sedimentation rate of $82 \mathrm{~mm} / \mathrm{h}$. Antiretroviral antibody was nonreactive. Antiretroviral serology and cytoplasmic antineutrophil cytoplasmic antibody were negative. None of the family members or neighbors had suffered from tuberculosis. He denied any past history of infectious diseases. The patient had Bacillus Calmette-Guerin vaccination scar.

Biopsy from the edge of the oral ulcer showed caseous epithelioid cell granulomas with acid-fast bacilli positivity [Figure 2a and b]. Periodic acid-Schiff

How to cite this article: Dey $B$, Deshpande $A H$, Bhat $\mathrm{SH}$, Singh A. Tuberculosis cutis orificialis with underlying pulmonary tuberculosis in an immunocompetent man. J Lab Physicians 2018;10:457-9. 
stain for fungus was negative. Fine-needle aspiration cytology from the Level II right cervical lymph node showed features of granulomatous lymphadenitis. A chest X-ray was advised, which showed cavitary changes in bilateral upper lungs with tree-in-bud nodules and patchy consolidation [Figure 3]. Sputum was positive for acid-fast bacilli. GeneXpert MTB/

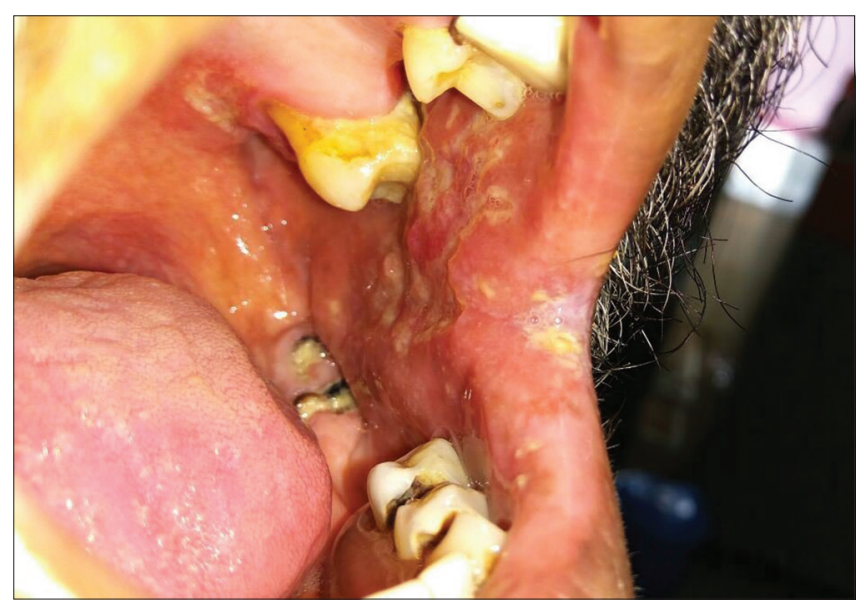

Figure 1: Ulcer in the right buccal mucosa extending to the right labial mucosa

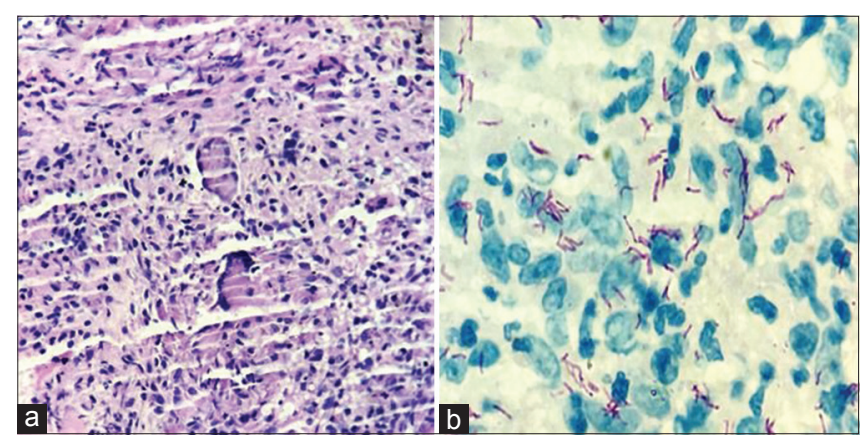

Figure 2: (a) Biopsy from the edge of the oral ulcer showed caseous epithelioid cell granulomas ( $\mathrm{H}$ and $\mathrm{E}, \times 100$ ). (b) Biopsy showing acid-fast positive bacilli (ZN Stain, $\times 1000)$

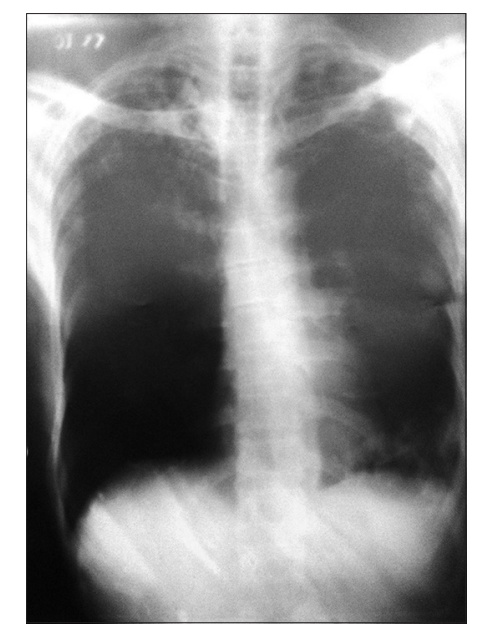

Figure 3: Chest X-ray showed cavitary changes in bilateral upper lungs with tree-in-bud nodules and patchy consolidation
RIF test based on nucleic acid amplification, which detects Mycobacterium tuberculosis-specific region of the rpoB gene and uses real-time polymerase chain reaction (RT-PCR), was done on sputum sample. The result was positive. The tuberculin skin test was strongly positive $(20 \mathrm{~mm} \times 25 \mathrm{~mm})$. A diagnosis of TCO in a case of pulmonary tuberculosis was made. The patient was treated with rifampicin, isoniazid, pyrazinamide, and ethambutol for the first 2 months. Then, rifampicin, isoniazid, and pyrazinamide were continued for further 4 months. There was a significant improvement in the ulceration within a month of treatment. After 6 months of therapy, there was complete healing of the oral ulcer, and chest $X$-ray showed the resolution of the lung lesions.

\section{Discussion}

TCO is a rare form of tuberculosis. It constitutes $2 \%$ of cutaneous tuberculosis cases and $0.01 \%-1 \%$ of all clinical presentations of tuberculosis. ${ }^{[1]}$ It usually occurs in immunosuppressed patients. ${ }^{[2]}$ It can be either primary or secondary. ${ }^{[1]}$ In primary TCO, the oral mucosa is the initial site of the infection without any internal organ involvement. ${ }^{[1]}$ Secondary TCO occurs in patients with internal organ tuberculosis due to autoinoculation of bacilli to the orificial area. ${ }^{[1]}$ Primary TCO usually affects children, while secondary oral involvement usually occurs in the middle and older age groups. ${ }^{[2]}$ The oral tuberculosis has myriad of manifestations and may present as ulcers nodules, fissures, or plaques and osteomyelitis of the jaw. It may present as single or multiple lesions. ${ }^{[2,3]}$

Secondary TCO of oral cavity is more often secondary to pulmonary tuberculosis. ${ }^{[3,4]}$ The most common site of involvement is tongue. Other sites of involvement include palate, buccal mucosa, and lips. ${ }^{[2,3]}$ Since most of the patients with TCO present with concomitant pulmonary tuberculosis, a chest X-ray is of paramount importance ${ }^{[5]}$ Although high-resolution chest computed tomography is superior in detecting pulmonary parenchymal disease, chest $X$-ray remains the first choice for evaluating pulmonary tuberculosis. ${ }^{[5]}$ Cavitation, which is found in up to $73 \%$ of pulmonary tuberculosis patients, is an important sign of tuberculous activity in lungs ${ }^{[6]}$ In the present case, the patient had presented with oral ulcer with concomitant pulmonary tuberculosis showing cavitation in chest $\mathrm{X}$-ray.

The differential diagnoses of TCO are deep fungal diseases, chronic traumatic ulcer, granulomatosis with polyangiitis, and squamous cell carcinoma. ${ }^{[3]}$ In the present case, biopsy established the diagnosis of $\mathrm{TO}$, which was confirmed by RT-PCR. About $95 \%-98 \%$ of multibacillary cases and $48 \%-53 \%$ of paucibacillary cases can be detected by culture and nucleic acid amplification 
tests. ${ }^{[2]}$ However, in $10 \%-20 \%$ of cases, the bacteriological confirmation is still not achieved, and in these cases, the diagnosis depends on the clinical or radiological improvement with treatment. ${ }^{[2,7]}$ Primary TCO is very rare as oral cavity is resistant to penetration of tubercular bacilli due to a thickness of the intact oral epithelium, cleansing action of saliva, local $\mathrm{pH}$, and antibodies present in saliva. ${ }^{[5,8]}$ Secondary TCO occurs due to oral inoculation with sputum or hematogenous spread of mycobacteria from another site ${ }^{[5]}$ In the present case, TCO may have developed due to oral inoculation or dissemination through hematogenous route.

Our case demonstrates that tuberculosis orificialis can manifest with concomitant pulmonary tuberculosis. Tuberculosis orificialis should be kept as a differential diagnosis of painful nonhealing oral ulcer.

\section{Declaration of patient consent}

The authors certify that they have obtained all appropriate patient consent forms. In the form the patient(s) has/ have given his/her/their consent for his/her/their images and other clinical information to be reported in the journal. The patients understand that their names and initials will not be published and due efforts will be made to conceal their identity, but anonymity cannot be guaranteed.

\section{Financial support and sponsorship} Nil.

\section{Conflicts of interest}

There are no conflicts of interest.

\section{References}

1. Turkmen M, Turk BG, Kandıloglu G, Dereli T. Tuberculosis cutis orificialis in an immunocompetent patient. Cutis 2015;95:E4-6.

2. Bhatia R, Mahajan R, Arava S, Singh S, Kandasamy D. A non-healing oral ulcer as a manifestation of systemic tuberculosis in an immunocompetent man. Indian J Dermatol Venereol Leprol 2017;83:238-41.

3. Chauhan V, Mahesh DM, Panda P, Mahajan S, Thakur S. Tuberculosis cutis orificialis (TBCO): A rare manifestation of tuberculosis. J Assoc Physicians India 2012;60:126-7.

4. Bayraktar K, Gürer G. Pulmonary tuberculosis presenting with oral aphthae. Eur J Rheumatol 2015;2:117-9.

5. Sun WL, Xu KL, Chen LL, Yu ZS. Tuberculosis cutis orificialis with both gingival involvement and underlying pulmonary tuberculosis. Aust Dent J 2011;56:216-20.

6. Lee JJ, Chong PY, Lin CB, Hsu AH, Lee CC. High resolution chest $\mathrm{CT}$ in patients with pulmonary tuberculosis: Characteristic findings before and after antituberculous therapy. Eur J Radiol 2008;67:100-4.

7. Frieden TR, Sterling TR, Munsiff SS, Watt CJ, Dye C. Tuberculosis. Lancet 2003;362:887-99.

8. Fujibayashi T, Takahashi Y, Yoneda T, Tagami Y, Kusama M. Tuberculosis of the tongue. A case report with immunologic study. Oral Surg Oral Med Oral Pathol 1979;47:427-35. 